Filipino Culture

\title{
Lohikang Makapamilya at Xiào (孝): Ang Etika sa Búhay Pamilya ng mga Filipino at Tsino
}

Noel L. Clemente

\begin{abstract}
The family plays a central role in the life of the Filipino that the interests of the state are sometimes compromised in favor of the interests of the family. This "family logic" of the Filipino culture is comparable to that of the ancient Chinese's xiào (孝) or filial piety. In one particular Analect, Confucius teaches that righteousness consists in the father concealing the misconduct of the son, and the son concealing the misconduct of the father. This portrays an apparently extreme adherence to filial piety, which goes against the interests of the state. In this paper, I explore how Confucianism explains and justifies this Analect, and whether the same reasoning could be applied to the Philippine setting.
\end{abstract}

Keywords: Confucianism, filial piety, family logic, concealment case

$\mathrm{M}$

ula sa mga dinastiya sa pamahalaan at pampamilyang negosyo, hanggang sa pagtulong sa mga malalayong kamag-anak at kahit ng mga ninong at ninang sa kasal, binyag, o kumpil, hindi maikakaila na nása pedestal ang pamilya sa búhay ng mga Filipino. Kadalasan, umaabot sa sukdulan itong pagkiling sa pamilya na hindi na naigagalang ang mga institusyon ng estado, tulad ng priyoridad na naibibigay sa mga kamag-anak ng mga doktor o abugado, at maging sa mga pampublikong opisina tulad ng SSS at NBI.

Hindi naman natatangì sa Filipinas ang ganitóng makapamilyang búhay. Sa kultura ng mga sinaunang Tsino, halimbawa, isa sa mga pinakamahalagang birtud ang xiào (孝, "filial piety") o paggalang sa pamilya. Umaabot din ito sa puntong nagkakatunggalian ang pamilya at estado, tulad ng sa isang kontrobersiyal na analekta tungkol sa pagnanakaw. Makikíta rito ang tensiyon sa pagitan ng ugnayang pampamilya at ugnayang pang-estado na umiiral sa Filipinas. 
Nilalayon ng papel na ito na tingnan ang nabanggit na pananaig ng pamilya sa estado sa pamumuhay ng mga Filipino at Tsino, at paghambingin ang dalawang kaso. Nais kong linawing bagaman panlipunang isyu ang tinatalakay, hindi ito isang sosyolohikal na pagsusuri, kundi isang pilosopikong paghahambing at pangangatwiran. Tutunghayan nitóng papel ang etikang nagbibigay-katwiran sa priyoridad na ibinibigay sa pamilya, upang mahinuha kung paano ito maaaring maging katanggap-tanggap sa lipunan.

Kaugnay nitó, mainam na linawing magkaiba ang saklaw ng lohikang makapamilya at ng xiào (孝)-nakatuon ang nahulí sa relasyon ng anak sa kaniyang mga magulang at ninuno, samantalang dumadaloy ang nauna sa lahat ng mga kamag-anak sa dugo, kasal, at iba pang apinidad. Gayunpaman, hindi sosyolohikal na paghahambing sa estrukturang pampamilya ng Filipinas at ng sinaunang Tsina ang paksa ng papel na ito, kundi ang etikal na pangangatwiran ng pagkiling sa pamilya, anupaman ang saklaw ng relasyong ito.

Ilalarawan ko sa unang bahagi ang "lohikang makapamilya" (hango kay Lukas Kaelin) na gumagabay sa búhay at pag-aasal ng mga Filipino at karaniwang nagbubunsod ng tensiyon sa kanilang estado. Sa ikalawang bahagi naman, ipapaliwanag ko ang xiào (孝) o "filial piety" ng mga sinaunang Tsino, pati na rin ang batayan nitó. Pagtutuunan ko rin ng pansin ang isang kontrobersiyal na analektang malinaw na nagpapakita ng tensiyon sa pamilya at estado, at ibibigay ko ang pangangatwiran ni Confucius at ng kaniyang mga iskolar. Sa ikatlo at hulíng bahagi, ilalantad ko ang pagkakaiba ng dalawang kultura, at ipapakita ko kung bakit hindi basta-basta maaaring ilapat ang pangangatwiran ni Confucius ng xiào (孝) sa sitwasyon ng makapamilyang lohika ng mga Filipino.

\section{Ang “Lohikang Makapamilya" sa Búhay ng mga Filipino}

Halos baság na ang kasabihang "pamilya ang pangunahing yunit ng lipunan," ngunit hindi eksaherado ang katotohanan nitó sa búhay ng mga Filipino, dahil ang ugnayan ng pamilya "ang balangkas ng lipunang Filipino." ${ }^{1}$ Ito ang nagsisilbing batayan ng pakikipag-ugnayan at katwiran ng mga mamamayan, at siya ring pundasyon ng mga institusyon, relasyon, pagpapahalaga, at pananaw sa búhay. ${ }^{2}$

${ }^{1}$ Salin ng may-akda ng "grand plan of Filipino society," mula sa F. Landa Jocano, Filipino Social Organization: Traditional Kinship and Family Organization (Quezon City: Punlad Research House, 1998), 11.

${ }^{2}$ Ibid., 13.

(C) 2017 Noel L. Clemente

http://www.kritike.org/journal/issue 20/clemente june2017.pdf

ISSN 1908-7330

(cc) BY-NC-ND 


\section{LOHIKANG MAKAPAMILYA AT XIÀO (孝)}

\section{Ang Ugnayang Pampamilya}

Nagsisimula ang ugnayang pampamilya sa batayang biyolohikal: ang pagiging magkadugo. Dahil namana ng isang anak ang dugo ng kaniyang ama at ina, nagiging "magka-dugo" ang anak at ang pamilya ng kaniyang mga magulang. ${ }^{3}$ Mayroon pang tinatawag na malapit at malayong kadugo; maituturing na malapit ang mga pamilya ng iyong mga magulang, at malayo naman ang mga kadugo ng mga kadugo. ${ }^{4}$

Nagiging lalong masalimuot ang konsepto ng pamilya sa pamamagitan ng kasal: nagiging magkaugnay ang dalawang pamilyang hindi magkadugo. Bago pa man magkaroon ng anak ang mag-asawa, nagkakaroon na ng mga panibagong ugnayan sa pagitan ng kanilang mga pamilya. ${ }^{5}$ Gayunpaman, mas mababa ang antas ng ganitóng ugnayan kaysa ugnayan ng mga magkakadugo. Maaari ring magkaroon ng bagong kapamilya sa pamamagitan ng pag-aampon. ${ }^{6}$ Nagkakaroon dito ng pagkakaiba sa biyolohikal na mga magulang (yaong nagpanganak) at ang mga magulang na umampon (yaong nag-aruga at nagpalaki).

Lalo pang lalawak ang saklaw ng pamilya sa pamamagitan ng pagiging ninong o ninang sa kasal, binyag, o kumpil. Kahit na hindi magkaugnay sa dugo o sa kasal, may batayang kultural naman ang ganitóng uri ng ugnayang pampamilya. ${ }^{7}$ Hindi na kasing-igting ng ugnayang magkadugo o mag-asawa ang ganitóng ugnayan, lalo na sa lungsod; bagaman nananatili sa pangalan ang pagiging ninong, ninang, at inaanak, hindi inaasahan ang malalim na pananagutan nila sa isa't isa. ${ }^{8}$

Samakatwid, malawak ang saklaw ng ugnayang pampamilya sa Filipinas; hindi lamang dugo at kasal ang batayan nitó, kundi pati mga kultural na ugnayan tulad ng pag-aampon at pagiging ninong o ninang. Ngayong nakita natin kung sino ang kinikilalang "kapamilya" ng mga Filipino, suriin naman natin ang epekto nitó sa kanilang pamumuhay.

${ }^{3}$ Ibid., 16. Nabubuo rin ang ugnayang magkadugo ng anak sa kaniyang mga kapatid, tito at tita, lolo at lola, at mga pinsan (kung mayroon).

${ }^{4}$ Makikita natin ang "antas" ng pagiging magkamag-anak sa ugnayang magpipinsan. "Pinsang buo" o "first cousin" iyong mga anak ng kapatid ng iyong mga magulang. "Pinsang pangalawa" o "second cousin" naman ang mga apo ng kapatid ng iyong mga lolo at lola. Meron ding antas ang mga desendiyente ("descendants"): "apo," "apo sa tuhod," "apo sa talampakan." Para sa mas detalyadong paliwanag, tingnan ang Jocano, Filipino Social Organization, 38-40.

5 Sa kasal, nagkakaroon ng "biyenan" (magulang ng asawa), "manugang" (asawa ng anak), "bayaw" (kapatid na lalaki ng asawa), "hipag" (kapatid na babae ng asawa), "bilas" (asawa ng kapatid), at "balae" (magulang ng asawa ng anak). Tingnan ang Jocano, Filipino Social Organization, 41.

${ }^{6}$ Jocano, Filipino Social Organization, 18.

${ }^{7}$ Ibid.

${ }^{8}$ Ibid., 45-46.

(c) 2017 Noel L. Clemente

http://www.kritike.org/journal/issue 20/clemente june2017.pdf

ISSN 1908-7330

(c) BY-NC-ND 


\section{Ang “Lohikang Makapamilya” sa Lipunan}

Malaki ang papel ng pamilya sa panahon ng kagipitan. Itinuturing na tungkulin at obligasyon ang pagtulong sa sinumang kamag-anak na nangangailangan. ${ }^{9}$ Kapag may nagkasakit o naospital, unang nilalapitan ang mga kapamilyang may koneksiyon sa ospital, o kayâ naman yaong mga makapagbibigay ng pantustos. Makikita rin nating ang pagsalalay sa pamilya sa mga kasal o búrol, kung saan dumadalo ang halos buong mag-anak para suportahan ang mga sangkot.10 Samakatwid, ang hindi pagtulong sa kapamilyang nangangailangan-kahit na napakalayong kamag-anak-ay itinuturing na paglalapastangan sa dangal ng pamilya. ${ }^{11}$

Sa ganitóng pananaw, lubhang mas mahalaga ang kapamilya kaysa sinumang estranghero.12 Ito ang tinatawag ni Kaelin na "lohikang makapamilya." 13 Paliwanag niya, upang maging panatag ang Filipino sa lipunan, sinusubukan niyang makipagkilala nang husto sa ibang tao, karaniwang batay sa pagiging magkatulad ng pinanggalingan, pinag-aralan o apelyido. Lalong panatag ang pakikipag-ugnayan kung maitatag ang ugnayang pampamilya sa pamamagitan ng mga batayang kultural. ${ }^{14}$ Samakatwid, nagkakaroon ng pagkiling sa mga itinuturing na "kapamilya," kadugo man o kaugnay lang bílang kumpare o inaanak. May pananagutan ang Filipino sa kaniyang mga kamag-anak - pananagutang hindi umiiral sa labas ng pamilya. ${ }^{15}$ Ang lipunang kinikilala ng isang indibidwal, sa gayon, ay isang lumalapad na ugnayang pampamilya. Dito rin natin makikita ang pamilya bílang "balangkas ng lipunang Filipino."

At sapagkat nagsisilbing batayan itong lohikang makapamilya ng paraan ng pakikiugnay sa ibang tao, masasabi nating pamilya ang batayan ng etikang Filipino. Karamihan ng mga pagpapahalagang Filipino, kabílang na ang pakikisama, hiya, at utang-na-loob, ay nakabatay sa ugnayan ng kapuwa, na sa huli'y nakabatay sa ugnayang pampamilya. Kayâ naman tinatangkilik ang pagiging maka-kapuwa at itinuturing na di-kanais-nais ang pagiging maka-sarili. ${ }^{16}$ Maramdamin ang mga Filipino kayâ naman

\footnotetext{
${ }^{9}$ Lukas Kaelin, Strong Family, Weak State: Hegel's Political Philosophy and the Filipino Family (Quezon City: Ateneo de Manila University Press, 2012), 99.

${ }^{10}$ Jocano, Filipino Social Organization, 57-58.

11 Ibid., 63.

12 Kaelin, Strong Family, Weak State, 103.

13 Salin ng may-akda mula sa "family logic." Mainam ang saling "makapamilya" upang bigyang-diin ang pagkiling ng mga Filipino sa kanilang mga kapamilya.

${ }^{14}$ Kaelin, Strong Family, Weak State, 102-103.

${ }^{15}$ Jocano, Filipino Social Organization, 155.

${ }^{16}$ F. Landa Jocano, Filipino Value System: A Cultural Definition (Quezon City: Punlad Research House, 1997), 8-9.
}

(C) 2017 Noel L. Clemente http://www.kritike.org/journal/issue 20/clemente june2017.pdf ISSN 1908-7330 


\section{8}

LOHIKANG MAKAPAMILYA AT XIÀO (孝)

"nakakahiya" kapag hindi ka nakikisama o nakakatulong sa kapuwa. ${ }^{17}$ Nakasalalay ang dangal ng Filipino sa pakikisama sa ibang tao: sa pakikipagkapuwa, pagmamalasákit sa kapuwa, at pagtanaw ng utang-naloob sa kapuwa. ${ }^{18}$

Gayunpaman, ang ganitóng pagkiling sa pamilya ay may katapat na pagpapaliban sa mas impersonal na aspekto ng lipunan. Dahil sa pagpapahalaga sa dangal ng pamilya, hindi natutuunan ng pansin ang mga abstraktong prinsipyo tulad ng katarungan sa lipunan. ${ }^{19}$ Klasikong halimbawa rito ang pagpapauna ng mga kapamilya sa pila, na karaniwang nangyayari sa ospital, sa panayam sa trabaho, o maging sa mga pagkuha ng mga lisensiya at ID. Dahil sa priyoridad na binibigay sa mga kapamilya, nalalabag ang ideya ng katarungan at pagkakapantay-pantay para sa mga nauna sa pila at naghintay nang mas matagal.

Lalong lumalalim itong problematikong pagkiling kapag estado na ang katunggali ng pamilya. Maging sa larangan ng politika at ekonomiya, inuuna pa rin ang pamilya-matingkad na halimbawa rito ang mga dinastiyang politikal at mga negosyong pampamilya. May kakaibang kakayahan ang ugnayang pampamilya na makalikha ng partidong politikal, na lalong maaaring lumaki sa pagsapi ng mga kaalyansang ibang pamilya. ${ }^{20}$

Makikita rin natin ang kapangyarihan ng isang pamilya sa estado sa tinatawag na "rent-seeking." Paliwanag ng ekonomistang si James Buchanan, ang rent ay "isang artipisyal na bentahang pang-ekonomiko na iginagawad ng estado sa pamamagitan ng pagtatakda ng hanggahan ng pagpasok sa merkado." 21 Isang magandang halimbawa nitó ang mga monopolyo: Kung Meralco lang ang nabigyan ng lisensiya ng pamahalaan upang magbenta ng kuryente, malaki ang kanilang kikitahin dahil wala silang katunggaling iba sa industriya. Sa gayon, nagkakaroon ng kompetisyon sa mga ganitóng rent, na tinatawag na rent-seeking. Sa ganitóng paraan, lumalago ang kapangyarihang pang-ekonomiko ng mga mayayamang oligarkiya, samantalang nawawalan ng kakayahan ang estado na magbigay ng mga serbisyong panlipunan, dahil nga naililipat sa mga pribadong kompanya (na pagmamay-ari ng mga oligarkiya) ang karapatan sa merkado. ${ }^{22}$ At dahil

${ }^{17}$ Ibid., 73.

18 Ibid., 82-83.

${ }^{19}$ Kaelin, Strong Family, Weak State, 104.

${ }^{20}$ Alfred W. McCoy, “'An Anarchy of Families': The Historiography of State and Family in the Philippines," in An Anarchy of Families: State and Family in the Philippines, ed. by Alfred W. McCoy (Quezon City: Ateneo de Manila University Press, 1993), 10.

${ }^{21}$ Salin ng may-akda, mula sa orihinal na "rents are created when a state gives an entrepreneur an artificial advantage by restricting 'freedom of entry' into the market." McCoy, "An 'Anarchy of Families,"' 11.

${ }^{22}$ Mas mauunawaan itong rent-seeking kung babalikan ang kasaysayan ng Republika ng Filipinas pagkatapos ng Ikalawang Digmaan Pandaigdig. Dahil naubos ang salapi ng

(c) 2017 Noel L. Clemente

http://www.kritike.org/journal/issue 20/clemente june2017.pdf

ISSN 1908-7330

(cc) BY-NC-ND 
nawawalan ng kapangyarihan ang estado, lalong umiigting ang relasyon sa loob ng pamilya, dahil kapag hindi nakatutulong ang estado o ang simbahan, sa pamilya lumalapit ang Filipino. ${ }^{23}$

Samakatwid, mahalagang pag-aralan at bigyang-katwiran ang lohikang makapamilya ng mga Filipino. Sa isang banda, ito ang nagsisilbing batayan ng pag-aasal ng mga Filipino sa lipunan-ito ang kanilang lohika. Ani Jocano, "sa anumang mangyari sa isang indibidwal, sangkot ang kaniyang buong pamilya." 24 Ngunit sapagkat makapamilya, nagkakaroon ng pagkiling sa pilîng mga tao at nakakaligtaan ang mas nakalalaking lipunan. Anuman ang pananagutan, obligasyon, at pagpapahalagang naikakabit sa mga kamag-anak ay hindi madaling makita sa ibang tao. ${ }^{25}$

Tulad ng nabanggit sa pambungad, hindi nag-iisa ang Filipinas sa pagkakaroon ng makapamilyang lipunan. Sa susunod na bahagi, titingnan naman natin ang pagkiling ng mga sinaunang Tsino sa pamilya, at kung paano nila kinakatwiranan ito.

\section{Ang Xiào (孝) sa buhay ng mga sinaunang Tsino}

Maihahambing ang lohikang makapamilya ng mga Filipino sa xiào ( 孝) o paggalang sa pamilya ng mga sinaunang Tsino. ${ }^{26}$ Isa ito sa mga pinakamahahalagang birtud para sa kanila, ayon sa mga pangaral ni Confucius. Itinuturing niyang xiào (孝) ang pinagmumulan ng ren (仁) o kagandahang-loob, na siyang pinakamahalagang birtud ng Confucianismo. ${ }^{27}$ Titingnan natin sa bahaging ito ang kasaysayan, katwiran, at kahalagahan ng xiào (孝) sa pamumuhay ng mga sinaunang Tsino. ${ }^{28}$

pamahalaan, kailangan nilang ibenta ang mga serbisyo sa mga makakapangyarihan at mayayamang mga pamilya. Lalong umigting ang pagbibigay ng mga rent noong panahon ng batas militar sa pamumuno ng dating pangulong Ferdinand Marcos; malaya niyang nabibigyan ng rent ang mga kakampi niyang oligarkiya upang makuha ang kanilang suporta. Tingnan ang McCoy, "An 'Anarchy of Families,'” 10-19.

${ }^{23}$ McCoy, "An 'Anarchy of Families,'” 7.

${ }^{24}$ My translation of "Whatever happens to a person involves his (a) buong mag-anak, (b) buong kamag-anak, and (c) buong angkan." Jocano, Filipino Social Organization, 159.

${ }^{25}$ Jocano, Filipino Social Organization, 157.

${ }^{26}$ Xiào is usually translated to "filial piety." The Chinese term, however, will be used for this paper.

27 Analects, 1:2. Maraming salin ang ren sa Ingles: "benevolence," "goodness," "righeousness" at iba pa.

${ }^{28}$ Nais kong bigyang-diin na pamumuhay ng mga sinaunang Tsino lang ang saklaw ng pagtalakay sa Xiào sa papel na ito. Bagaman maigting pa rin ang impluwensiya marahil ng pamilya sa buhay ng mga kontemporanyong Tsino, hindi maikakailang sa pamumuhay ng mga sinaunang Tsino makikita ang ugat at sukdulan ng birtud ng Xiào.

(C) 2017 Noel L. Clemente

http://www.kritike.org/journal/issue 20/clemente june2017.pdf

ISSN 1908-7330

(cc) BY-NC-ND 


\section{LOHIKANG MAKAPAMILYA AT XIÀO (孝)}

\section{Kasaysayan at Panimulang Katwiran ng Xiào (孝)}

Masasabing nagsimula ang birtud na xiào (孝) sa relihiyosong pagsamba sa mga ninuno. Nakakalap ang arkeologong si Chang Kwang-chih ng katibayan sa ganitóng pagsamba sa mga prehistorikong Tsino. ${ }^{29} \mathrm{Sa}$ katunayan, dumadalangin sila sa kanilang Diyos na si Shangdi sa pamamagitan ng pagdarasal sa mga ninuno ng emperador. ${ }^{30}$ Kalaunan, naisalin ang pagsamba sa mga ninunong yumao sa paggalang at pagmamahal sa mga magulang na nabubuhay pa. Itinuturing pa ngang sukdulang krimen sa panahon ng dinastiyang Chou ang paglabag sa mga utos ng magulang. ${ }^{31}$

Isa si Confucius sa mga tagapagdakila ng xiào (孝) sa búhay ng mga sinaunang Tsino. Bagaman pinakamahalagang birtud pa rin ang ren (仁) sa kaniyang pagtuturo, malaking papel pa rin ng pagiging mabuting anak sa pagkakaroon ng mabuting búhay. Ipinapaliwanag ni Hsieh Yu-Wei na "Likás ang pagmamahal ng tao sa kaniyang mga magulang, tulad ng mga paslit na hindi na inaaral mahalin ang kanilang ama't ina. Sa pagmamahal na ito (xiào (孝)) nagbubunsod ang kagandahang-loob. Ito ang unang hakbang sa tumutubong birtud ng pag-ibig sa sangkatauhan."32 Sapagkat hindi natututuhan ang pagmamahal sa magulang, di tulad ng pag-ibig sa bayan o sa mga kaibigan, angkop lang na ituring ang xiào (孝) bílang unang hakbang tungo sa ren (仁).

Dahil sa pagpapahalagang ito ng mga sinaunang Tsino sa xiào (孝), hindi nakagugulat na pamilya ang nása kaibuturan ng kanilang lipunan. Ayon sa The Great Learning, kailangan munang maalagaan ang pamilya bago mapamahalaan nang maayos ang estado. ${ }^{33}$ Ang katwiran ng mga pagtuturo, imposibleng maturuan ang iba kung hindi mo kayang turuan ang sarili mong pamilya. ${ }^{34}$ Samakatwid, ang estado ay itinuturing lámang na pinalaking

\footnotetext{
${ }^{29}$ Donald Holzman, "The Place of Filial Piety in Ancient China," in Journal of the American Oriental Society, 118:2 (April-June 1998): 185.

${ }^{30}$ Ibid., 186.

${ }^{31} \mathrm{Ibid} ., 188$.

32 Salin ng may-akda mula sa orihinal na: "[H]uman love towards parents is innate, as in the case of little children, without any need of being acquired through study. This inherent love-and-respect toward parents is the feeling of filial piety. And this is the fountainhead of benevolence. It is the germination or starting point of the gradually expanding virtue, the universal love of mankind." Hsieh Yu-Wei, "Filial Piety and Chinese Society," in The Chinese Mind: Essentials of Chinese Philosophy and Culture, ed. by Charles A. Moore (Honolulu: University of Hawaii Press, 1967), 170.

${ }_{33}$ Ang ginamit na sanggunian para sa The Great Learning ay Confucius, The Great Learning, in A Source Book in Chinese Philosophy, trans. by Wing-tsit Chan (New Jersey: Princeton University Press, 1963), I.4; pp. 85-94.

34 The Great Learning, IX.1.
}

(c) 2017 Noel L. Clemente

http://www.kritike.org/journal/issue 20/clemente june2017.pdf

ISSN 1908-7330

(c) BY-NC-ND 
pamilya: ang pinuno ang ama; ang mga mamamayan ang anak. At hangga't napapanatili ang kaayusan sa pamilya, magiging maayos din ang estado.

Sa kabila ng kahalagahan ng xiào (孝) sa etika ni Confucius, hindi nitó ibig sabihin na sinasaklaw nitó ang ibang birtud bílang pinakamahalagang prinsipyo. Kailangang pakatandaan na hakbang lámang ang xiào (孝) patungo sa ren (仁) na siyang sukdulang layon ng búhay-etikal. Kayâ naman sa mainam na kasabay ng paghubog ng xiào (孝) ay nililinang din ang ibang mga birtud. ${ }^{35}$

Gayunpaman, may mga iskolar na tumutuligsa sa labis na pagpapahalaga ng mga Tsino sa xiào (孝). May mga pagkakataong nasasakripisyo ang ibang mga birtud ng pagpapakatao sa taimtim na pagpapanatili ng xiào (孝). Maisasakongkreto natin itong tensiyon na ito sa pagtalakay sa isang kontrobersiyal na anale kta ni Confucius.

\section{Ang Katwiran ng Analekta 13:18}

\section{Ayon sa Analekta 13:18:}

Sinabi ng duke ng Sheh kay Confucius, "Sa bayan namin, may mga itinuturing kaming matutuwid na tao. Kapag nagnakaw ng tupa ang ama nila, isinusumbong nila sa kinauukulan." Tugon ni Confucius, "Sa bayan namin, iba ang kinikilalang matuwid. Pinagtatakpan ng mga ama ang kamalian ng kanilang anak, at pinagtatakpan ng anak ang kamalian ng kanilang ama. Ito ang katuwiran. ${ }^{36}$

Sa analektang ito, tíla isinusulong ni Confucius na nararapat pagtakpan ng ama at anak ang kamalian ng isa't isa. Ipinapakita ng analekta ang maaaring tensiyon na maidulot ng matapat na pagpapanatili ng xiào (孝 ). May mga pagkakataong magtatalaban ang xiào (孝) at birtud ng, sa halimbawang ito, katapatan o paggalang sa estado, at ayon kay Confucius, nananaig ang pagpapahalaga sa pamilya.

Hindi naman sa sinasabi ni Confucius na tama ang magnakaw, ngunit ganoon lang kaigting ang ugnayan ng magkakamag-anak na sa ganitóng mga pagkakataon ng tensiyon, kailangan pa ring unahin ang xiào (

${ }^{35}$ Hsieh Yu-Wei, "Filial Piety and Chinese Society," 173-174.

36 Salin ng may-akda ng Analekta 13:18. Mula sa puntong ito, gagamitan ko ang "analekta" para tukuyin itong partiular na analektang ito. Ang ginamit na sanggunian para sa mga susunod na analekta ay Confucius, Analects, trans. by Edward Slingerland (Indianapolis: Hacket Publishing Company, Inc., 2003). 


\section{LOHIKANG MAKAPAMILYA AT XIÀO (孝)}

孝). ${ }^{37}$ Ganoon na lang kaeksaherado ang priyoridad sa xiào (孝) na, ayon sa pagsusuri ni Qingping Liu, tinuturo ni Confucius na kailangang unahin ang ugnayang pampamilya kaysa sa mga mas mataas na prinsipyo ng pagpapakatao. 38

Hindi nalalayo ang kaso ng analekta sa nangyayaring tensiyon sa pamilya at estado sa buhay ng mga Filipino. Kayâ naman, mainam na tingnan kung paano binibigyang-katwiran ng etika ni Confucius ang pagiging matuwid ng pagkukubli ng kamalian ng kamag-anak. Inilatag nina Lijun Bi at Fred D'Agostino ang mga tensiyon sa Confucianismong lumalantad sa kasong nabanggit. Tatalakayin ko ang dalawa sa mga ito. ${ }^{39}$

\section{Ming (名) at fen (分)}

Kinikilala ng Confucianismo ang pagkakaiba ng posisyon at papel ng mga tao sa lipunan. Tinuturo ni Confucius na magkakaroon ng kaayusan kung bawat tao ay lulugar sa kani-kaniyang posisyon (ming 名, na nangangahulugang "pangalan") at gagampanan ang kani-kaniyang tungkulin (fen 分, na nangangahulugang "papel") sa lipunan. May matalik na ugnayan ang ming (名) at fen (分): bawat ming (名) ay may tinutukoy na tiyak na fen (分). Sa gayon, tungkulin ng ama, bílang punò ng pamilya, na magsilbing huwaran sa kaniyang mga anak. Ngunit sa kaso ng analekta, hindi mabuting halimbawa ang pagnanakaw ng ama, kayâ hindi niya nagagampanan ang kaniyang fen (分) bílang ama. Para naman sa isang anak, tungkulin niyang sundin at igalang ang kaniyang ama. Ngunit paano niya bibigyang-dangal ang ama kung pagtatakpan niya ang kaniyang pagnanakaw? ${ }^{40}$

Gayunpaman, nararapat na ikubli ng anak ang kamalian ng ama dahil iyon ang kaniyang fen (分) bílang anak. Ganoon kalaki ang kahalagahang ibinibigay ni Confucius sa ugnayang mag-ama (o mag-ina);

\footnotetext{
${ }^{37}$ Benjamin I. Schwartz, The World of Thought in Ancient China (Cambridge: The Belknap Press of Harvard University Press, 1985), 103.

${ }^{38}$ Acoording to Qingping Liu, "one should place blood ties above 'higher' ideals of humanity, even to the point of abandoning these higher ideals if their realization obstructs or endangers the practice of filial piety and brotherly duties." Tingnan ang Qingping Liu, "Filiality versus Sociality and Individuality: On Confucianism as 'Consanguinitism'," in Philosophy East and West, 53:2 (April 2003): 236.

${ }^{39}$ Nagbigay ng apat na tensiyon na makikita sa kaso ng Analekta 13:18 sina Bi at D'Agostino. Ang una ay nakatuon sa pagsamba sa mga ninuno at sa ugnayan ng ama at anak. Ang ikatlo ay tungkol ay pagtalima sa mga magulang. Bagaman may mahihinuha tayo sa pagsiyasat sa mga tensiyong ito, mas malapit ang ikalawa at ikaapat na talaban sa pagtupad sa pakay ng papel na ito. See Lijun Bi at Fred D'Agostino, “The Doctrine of Filial Piety: A Philosophical Analysis of the Concealment Case," in Journal of Chinese Philosophy, 31:4 (December 2004): 451-467.

${ }^{40}$ Ibid., 453.
}

(c) 2017 Noel L. Clemente

http://www.kritike.org/journal/issue 20/clemente june2017.pdf

ISSN 1908-7330

(cc) BY-NC-ND 
kahit na hindi mabuting halimbawa ang ama, kailangan pa ring tupdin ng anak ang kaniyang fen (分). ${ }^{41}$

Bílang paglalagom, itinuturo ng doktrina ng ming (名) at fen (分) na kailangang kumilos ayon sa papel at tungkulin sa estado upang maging matiwasay ang pamumuhay sa lipunan. Tungkulin ng anak, bílang anak, na igalang ang kaniyang mga magulang, at panatilihin ang mabuti nilang ugnayan. Sa kaso ng analekta, kailangan niya pa ring tupdin ang kaniyang fen (分) bílang anak, kahit ang ama niya mismo ay tumataliwas sa kaniyang fen (分) bílang ama.

Nais kong punahin na hindi naisasaalang-alang nitóng doktrina ng ming (名) at fen (分) na maaaring magkaroon ng higit sa isang ming (名) at fen (分), dahil marami táyong ginagampanang papel sa lipunan. Halimbawa, ang anak ay isa ring mamamayan ng estado, at mapapansing may tunggalian sa pagtupad sa kaniyang fen (分) bílang anak at sa kanyang fen (分) bílang mamamayan. Tutunghayan natin ito sa susunod na mga talata.

\section{Xiào (孝) at katapatan sa estado}

Tulad ng nabanggit, itinuturing ni Confucius na ekstensiyon ng pamilya ang estado. Ibig sabihin, ang paggalang at pagmamahal sa pamilya mula sa xiào (孝) ay nararapat na ibaling din sa lipunan. Ngunit sa kaso ng analekta, nagkakaroon ng tunggalian sa katapatan ng anak-hinihingi ng xiào (孝) na huwag niyang hayaang mapahamak ang kaniyang ama dahil sa pagnanakaw nitó, ngunit bílang alagad ng pinuno ng estado, tinatawag din siyang maging tapat sa batas at ilantad ang anumang krimen, tulad ng pagnanakaw ng kaniyang ama. ${ }^{42}$ Lalong lumalalim ang tensiyon kung isasaalang-alang nating ang xiào (孝) ang pundasyon ng katapatan sa estado. Samakatwid, ang katapatan ng anak sa ama ang pinagmumulan ng katapatan niya sa estado, ngunit sa kaso ng analekta, ang katapatan sa isang panig ay pagtataksil sa isa pa.

Natutugunan ang tunggaliang ito sa pagbibigay-priyoridad sa xiào ( 孝) sa kasong ito, dahil sa xiào (孝) mismo nakasalalay ang katapatan sa estado. Sapagkat sa ugnayang pampamilya ang huwaran ng magandang ugnayang pampolitika sa estado, maituturing din na may aspektong politikal ang ugnayan ng ama at anak. Nilalayon ng Confucianismo na itaguyod ang kaayusan sa pamilya nang maging maayos din ang estado. Samakatwid, walang argumento sa doktrina ng Confucianismo na tumatangkilik sa tunggalian ng xiào (孝) at katapatan sa estado. ${ }^{43}$

Sa kabila nitó, hindi natin matatakasan ang tensiyon sa dalawang fen (分) ng anak sa analekta. Maaaring makatulong sa puntong ito ang

${ }^{41}$ Ibid., 457.

42 Ibid., 455.

${ }^{43}$ Ibid., 464.

(C) 2017 Noel L. Clemente http://www.kritike.org/journal/issue 20/clemente june2017.pdf ISSN 1908-7330 


\section{LOHIKANG MAKAPAMILYA AT XIÀO (孝)}

pagtatambis sa dalawang uri ng pagkukubli sa krimen ng ama: Maituturing na aktibong pagkukubli ang pagsisinungaling kapag tinanong tungkol sa krimen, at pasibong pagkukubli naman ang pagnanasàng huwag mabuking ang ama. ${ }^{44}$ Kung tutuusin, halos likás naman sa kahit na sinong anak na naising huwag maparusahan ang kaniyang ama, kahit na mali ang kaniyang nagawa (pasibo). ${ }^{45}$ Ngunit ibang usapan ang pagsisinungaling at pagtangging may kaalaman sa nasabing krimen kapag tinanong na ng hukom (aktibo). Samantalang mahalaga ang ugnayang pampamilya kayâ maaaring unahin ito kaysa ang pagsunod sa pinuno ng estado, may hanggahan din ang priyoridad na ito. Sa kasong ito, katanggap-tanggap ang pasibong pagkukubli sa krimen ng ama, ngunit mali na ang aktibong pagkukubli, sapagkat lumalabis na ang pagsasawalang-bahala sa awtoridad ng estado.

Bílang paglalagom, may tuksong akalaing itinuturo ng analekta na maaaring labagin ang batas alang-alang sa pamilya. Ngunit ang pinakapunto ni Confucius ay pamilya ang batayan ng lipunan, at sa xiào (孝) nagsisimula ang ren (仁). ${ }^{46}$ Kailangang tandaang kapuwa mahalaga ang tungkulin sa pamilya at tungkulin sa estado. Sa mga pagkakataong magkakaroon ng tensiyon sa kanila, kailangang suriin ang sitwasyon: kung makatwiran bang paboran ang isa. Sa kaso ng analekta - na maaaring hindi totoo sa ibang mga sitwasyon-makatwiran ang pasibong pagkukubli upang mapanatili ang mabuting ugnayan ng mag-ama, ngunit hindi na katanggap-tanggap ang aktibong pagkukubli dahil hindi na naigagalang ang estado. Pagkatapos ng lahat, "Iginigiit ng turo ni Confucius na ang anak na may xiào (孝) ay hindi kikilos nang labag sa estado sa huli't huli, kahit na mapilitan siyang lumabag sa ilang natatanging pagkakataon." 47

\section{Ang Etika ng Makapamilyang Lohika at Xiào (孝)}

Ngayong napagmasdan na natin ang sitwasyon ng pamilyang Filipino at pilosopiya ng pamilyang Tsino, susubukin nating ilapat ang katwiran ni Confucius sa suliranin ng lohikang makapamilya. Ngunit bago iyon, kailangan munang paghambingin at pagtambisin ang dalawang kultura pagdating sa pamilya.

${ }^{44}$ Ibid., 465 .

${ }^{45}$ Ibid., 458 .

46 Manuel B. Dy, Jr., "Confucian Ethics in Modern Society: Appropriating Confucianism in Contemporary Discourses," in Prajñā Vihāra: Journal of Philosophy and Religion, 13:1-2 (2012): 89.

47 Salin ng may-akda mula sa "Confucianism insists that if filial piety is well established in the family, then the family would not produce a member who would act contrary to the interests of the state in the long run, even if he is required to do so in specific cases." Hsieh Yu-Wei, "Filial Piety and Chinese Society," 465.

(c) 2017 Noel L. Clemente

http://www.kritike.org/journal/issue 20/clemente june2017.pdf

ISSN 1908-7330

(c) BY-NC-ND 


\section{Makapamilyang Lohika vs. Xiào (孝)}

Una sa lahat, mapapansing magkahawig ang pinagmumulan ng pagkakahalal ng pamilya sa pedestal sa dalawang kultura-kapuwa pamilya ang batayan ng pamumuhay sa lipunan. Ang ugnayang pampamilya ang panimulang karanasan ng mga Filipino sa pakikisalamuha sa ibang tao, kayâ mahalaga para sa kanilang magtatag ng relasyong mala-pamilya man lang para maging panatag sa pakikiugnay. Samantala, itinuturing naman ng mga sinaunang Tsino na unang hakbang ang xiào (孝) para sa napakahalagang birtud ng ren (仁), na lumalayon sa pag-ibig sa sangkatauhan.

Bagaman parehas silang sasang-ayon na ang lipunan ay pamilyang pinalaki lang, may pagkakaiba sa kanilang pangangatwiran. Sa mga Filipino, batayan ang pamilya ng ugnayan at pakikisalamuha; sa mga sinaunang Tsino, batayan ito ng paglinang sa kagandang-loob o ren (仁). Samakatwid, etikal ang motibasyon ng mga Tsino sa xiào (孝), samantalang hamak na pamumuhay at pakikiugnay ang pinanggagalingan ng lohikang makapamilya ng mga Filipino.

Maaari pa tayong mangahas na sabihing magkasalungat ang ugnayan ng etika at pamilya sa dalawang kultura. Nakita natin kanina na pamilya ang batayan ng etikang Filipino-Ang maituturing na "tamang kilos" o "magandang asal" ay yaong magpapanatili ng mabuting ugnayang pampamilya. Samantala, para sa mga sinaunang Tsino, alinsunod sa Confucianismo, ang kahalagahan ng ugnayang pampamilya ay nakabatay sa etikal-Mahalaga ang xiào (孝) dahil ito ang unang hakbang tungo sa ren (仁 ), na siyang lalong mahalaga. Sa madaling sabi, ang makapamilyang lohika ng mga Filipino ang batayan ng kanilang etika, samantálang etika ang batayan ng xiào (孝) ng mga Tsino.

Ngayong tumambad na ang kaibhan ng relasyon ng etika sa ugaling pampamilya sa dalawang kultura, isaalang-alang natin sa mga susunod na talata na may hanggahan ang bisà ng paglapat ng katwirang Tsino sa sitwasyong Filipino.

\section{Ang Katwiran ng Xiào (孝) para sa Lohikang Makapamilya}

Ang solusyong nakuha natin sa tensiyon ng analekta ay ang pagkakaiba ng aktibong pagkukubli at pasibong pagkukubli ng kamalian ng ama. Mahalagahang panatilihin ang mabuting relasyon ng ama at anak dahil ito ang pundasyon ng kagandahang-loob, ngunit may hanggahan ang priyoridad na naibibigay sa xiào (孝) kaysa sa katapatan sa estado. Sa gayon, pasibong pagkukubli lámang ang katanggap-tanggap.

Sa kaso ng pamilyang Filipino, isusulong din ng makapamilyang lohika na ang pananagutan sa pamilya ang dapat unahin. Muli, may 


\section{LOHIKANG MAKAPAMILYA AT XIÀO (孝)}

pagkakatulad at pagkakaiba itong kaisipang Filipino at ang katwiran sa Analekta 13:18. Sa isang banda, layunin din ng isang Filipino na panatilihin ang magandang ugnayan sa loob ng pamilya. Sa kabilâng banda, halos hindi kinikilala ng Filipino ang estado, kaiba sa pagpapahalagang ibinibigay ng mga sinaunang Tsino sa kanilang gobyerno. Samakatwid, kung mararanasan ng isang Filipino ang sitwasyon sa analekta, kung magnakaw ng tupa (o kung anuman) ang kaniyang ama, marahil wala na siyang pag-aalinlangan sa pagkubli-parehas na pasibo at aktibong pagkukubli-sa kamalian ng ama. Halos absoluto ang makapamilyang lohika sa mga Filipino, dahil nga ito ang batayan ng etika.

Lumilitaw rito ang hanggahan ng paglapat ng katwiran ng xiào (孝) sa sitwasyon ng mga Filipino. Tandaang may batayang etikal ang pagpapahalaga ng mga Tsino sa pamilya, samantalang baligtad (pamilya ang batayan ng etika) sa kaso ng mga Filipino. Layunin ng Confucianismo na linangin ang ren (仁) o kagandahang-loob, na sa pamilya nagsisimula, at kalauna'y nararanasan sa estado. Ngunit sa kaso ng mga Filipino, pamilya ang nagsisilbing pundasyon ng pakikiugnay sa lipunan, na lumilikha ng etika na hindi parating makatwiran.

\section{Pangwakas}

Samakatwid, maiuuwi ang suliranin ng lohikang makapamilya ng mga Filipino sa kawalan ng motibasyong etikal ng ugnayang pampamilya. Makatwiran ang xiào (孝) ng mga sinaunang Tsino dahil nakabatay ito sa paglaláyong maatim ang birtud ng ren (仁)-May batayang etikal ang ugnayang pampamilya. Samantala, hindi tumatalab ang ganitóng katwiran sa lohikang makapamilya ng mga Filipino sapagkat wala itong pundasyong etikal; sa halip, ang ugnayang pampamilya pa nga ang batayan ng kanilang etika.

Layon ko lámang ipaliwanag ang etikal na katwiran-o tulad ng naipakita ko, kawalan ng katwiran-ng lohikang makapamilya. Ibang pananaliksik na ang pagmumungkahi ng kongkretong hakbang tungo sa pagtutuwid ng ganitóng estruktura ng etika sa mga Filipino. ${ }^{48}$

Department of Philosophy, Ateneo de Manila University, Philippines

\footnotetext{
${ }^{4}$ Nagpapasalamat ako kay Dr. Manny Dy sa kaniyang kurso sa Kasaysayan ng Sinaunang Pilosopiyang Tsino na pinagmulan ng mga ideyang isinulat ko sa papel na ito, at kay Iman Tagudiña sa kaniyang tulong sa pagwawasto ng estilo ng manuskrito.

(C) 2017 Noel L. Clemente http://www.kritike.org/journal/issue 20/clemente june2017.pdf ISSN 1908-7330

(cc) BY-NC-ND
} 


\section{References}

Confucius, Confucius, Analects, trans. by Edward Slingerland (Indianapolis: Hacket Publishing Company, Inc., 2003).

The Great Learning, in A Source Book in Chinese Philosophy, trans. by Wing-tsit Chan (New Jersey: Princeton University Press, 1963).

Dy Jr., Manuel B., "Confucian Ethics in Modern Society: Appropriating Confucianism in Contemporary Discourses," in Prajñā Vihāra: Journal of Philosophy and Religion, 13:1 (2012).

Holzman, Donald, "The Place of Filial Piety in Ancient China," in Journal of the American Oriental Society, 118:2 (1998).

Hsieh Yu-Wei, "Filial Piety and Chinese Society," in The Chinese Mind: Essentials of Chinese Philosophy and Culture, ed. by Charles A. Moore (Honolulu: University of Hawaii Press, 1967).

Jocano, F. Landa, Filipino Social Organization: Traditional Kinship and Family Organization (Quezon City: Punlad Research House, 1998).

Filipino Value System: A Cultural Definition, (Quezon City: Punlad Research House, 1997).

Kaelin, Lukas, Strong Family, Weak State: Hegel's Political Philosophy and the Filipino Family (Quezon City: Ateneo de Manila University Press, 2012).

Lijun Bi and Fred D'Agostino, "The Doctrine of Filial Piety: A Philosophical Analysis of the Concealment Case," in Journal of Chinese Philosophy, 31:4 (December 2004).

McCoy, Alfred W., "'An Anarchy of Families': The Historiography of State and Family in the Philippines," in An Anarchy of Families: State and Family in the Philippines, ed. by Alfred W. McCoy (Quezon City: Ateneo de Manila University Press, 1993).

Qingping Liu, "Filiality versus Sociality and Individuality: On Confucianism as 'Consanguinitism'," in Philosophy East and West, 53:2 (April 2003).

Schwartz, Benjamin I., The World of Thought in Ancient China (Cambridge: The Belknap Press of Harvard University Press, 1985). 